\title{
Intereses y tendencias de la educación musical hoy: un estudio a partir de sus revistas científicas
}

\section{Amparo Porta}

Universitat Jaume I. Castellón. España. porta@edu.uji.es

\begin{abstract}
Resumen
La difusión de la educación musical es el lugar público de encuentro de la innovación y la investigación, y por ello su mejor escaparate científico y una sintesis de sus aspiraciones, aportaciones y tendencias, sin embargo, se encuentran pocos trabajos que la estudien. Este artículo se interesa por la especificidad de la educación musical, sus relaciones con la interdisciplinariedad y también sus diferentes aproximaciones metodológicas como elemento determinante. El objetivo es analizar sus intereses en revistas científicas especializadas y artículos publicados en una muestra. Para ello se han estudiando las palabras clave de los artículos seleccionados y líneas editoriales de las revistas a que pertenecen. Se han realizado 3 análisis de contenido a 7 revistas nacionales e internacionales de Educación musical y analizando 148 artículos para conocer sus dominios, tendencias, especificidad y aproximaciones metodológicas. Los resultados dan cuenta de los temas de interés destacados de las revistas y también de sus autores, mostrando así sus cuatro grandes áreas temáticas: Investigación, Formación, Contenidos y Práctica Musical como elementos centrales de sus intereses. El artículo termina mostrando, un estudio de dispersión y líneas de tendencias señalando la posición tanto de los autores como de las revistas en este juego de fuerzas que cobran visibilidad y sentido al establecer comparaciones entre ellas.
\end{abstract}

Palabras Clave: : Intereses, revistas, palabras clave, líneas editoriales

\section{Introducción}

La difusión de la educación musical es el lugar público de encuentro de la innovación y la investigación, y por ello su mejor escaparate científico. Este espacio supone, igualmente, una síntesis de las aspiraciones de los trabajos realizados, proyectos y tendencias, y la razón 
de que haya sido elegido como base de este trabajo. Desde el ámbito no formal e informal, la educación musical toma su sentido e impulso de la importancia que la sociedad concede a la música en la vida diaria y erudita, cultural, patrimonial, industrial y comunicativa, de las que siempre es un reflejo (Porta, 2017). Su carácter identitario crea manifestaciones de muy diferente índole en las colectividades, así como conexiones con diferentes áreas de conocimiento, y, como manifestación de la vida diaria, su presencia es incuestionable en la sociedad y el hábitat. Sin embargo, encontramos pocos trabajos que aborden su difusión como espacio de visibilidad, aspiraciones y tendencias en investigación e innovación, tanto del campo como en sus relaciones y conexiones con el resto de la comunidad científica. Y este hecho conviene tenerlo en cuenta porque dificulta la valoración de sus necesidades y aspiraciones dentro del espacio nacional, europeo y mundial de la Educación. El sistema por competencias de la educación supone el primer filtro con una apuesta clara de la Música en los niveles obligatorios por la interdisciplinariedad, que tiene como asignatura pendiente a día de hoy, una revisión en profundidad de las relaciones entre contenidos interdisciplinares y singularidades de la Música. Esta falta de definición del propio campo crea controversia entre los términos especificidad e interdisciplinariedad en ocasiones, debido a las dos competencias generales implicadas: la competencia lingüística y la competencia artística, cultural y expresiva (Porta, 2016). Los elementos de máxima especificidad de la música son su propio lenguaje (Sloboda, 1985; Porta, 2077) y los componentes técnicos de la interpretación musical en los niveles profesionales que requieren de métodos mixtos para poder ser visibilizados en sus foros, divulgados en sus espacios públicos y comprendidos como campo científico

El objetivo de este trabajo es analizar los intereses de la educación musical a partir de una muestra de revistas científicas de Educación Musical y sus artículos publicados en 2015 y 2016.

\section{Revisión de la literatura}

\subsection{Palabras clave}

Las palabras clave (a partir de ahora PC) surgen como un producto de internet como indexadores y motores de búsqueda. Sin embargo, además de estos usos, existen otros de índole académico que afectan a la selección de contenidos y preferencias. Desde la óptica de la difusión científica, las palabras clave son a modo de descriptores que no llegan a tener un vocabulario controlado como los Thesaurus -entre los que destaca en nuestro campo el de la UNESCO- que presentan una lista estructurada de términos para el estudio temático y 
búsqueda de documentos en múltiples campos (Tesauro de la Unesco, 1984). E igualmente, otro elemento de interés en nuestro estudio como sistema clasificatorio es la norma ISO (International Organization for Standardization) (Dextre Clarke \& Zeng, 2012; ISO 2788, 1986), en la que se define un indicador como «una expresión (numérica, simbólica o verbal) utilizada para caracterizar actividades (sucesos, objetos, personas) en términos cuantitativos y cualitativos, con el propósito de evaluar el valor de las actividades caracterizadas y el método asociado» (Martínez Tamayo, 2011, p. 32).

\subsection{Las revistas y las líneas editoriales}

Para el estudio de las revistas de educación musical se ha buscado una muestra significativa de las publicaciones. Se han revisado revistas que figuran en el Arts \& Humanities Citation Index; el Social Sciences Citation Index y Scopus, 39 que figuran en el Catálogo de Latindex y Scielo y, finalmente, 18 que poseen referato pero no aparecen en los índices anteriores. A partir de la revisión inicial se recopilaron 86 revistas que no lo agotan y revisado 29 para, finalmente, centrarnos en 7 de ellas que pudieran ser comparables. Todas ellas disponen y hacen públicas sus líneas editoriales (a partir de ahora LE), tienen intereses centrados en la educación musical tanto de ámbito internacional como españolas, están clasificadas como de alto y medio impacto y disponen de comités de selección y referees que requieren de dos votos favorables de tres posibles para la publicación de los artículos.

\section{Método}

\subsection{Instrumentos utilizados y diseño de la investigación}

Se ha utilizado una metodología mixta que ha tenido como principal instrumento el análisis de contenido mediante nubes de palabras y análisis de texto con soporte informático con los programas MxQda y Atlas.ti. En esta investigación se estudiaron los artículos, PC y LE de siete revistas de los años 2015 y 2016. De ellas, cuatro aparecen en la base de datos de la Web of Science (ISI) estando tres en el JCR y una en Scopus, mientras, el resto, todas las españolas, en el periodo indicado no estaban incluidas en ellas. Se realizaron tres análisis de contenido a todos los artículos y revistas seleccionadas para estudiar las PC, las LE y su comparaciones en dominios, especificidad así como aproximaciones analíticas y metodológicas. Nuestro foco se centra en conocer qué es lo que interesa actualmente saber 
y explorar en la educación musical, y de que manera estos artículos se aproximan a sus temáticas e interrogantes.

\subsection{Las palabras clave y las líneas editoriales}

El procedimiento general ha sido la selección de las palabras, su posterior codificación y creación de categorías por el sistema inductivo basado en los datos de la Grounded Theory (Strauss \& Corbin, 1990). Se han seleccionado las correspondientes a 148 artículos obteniendo 424 códigos que fueron agrupados de manera progresiva hasta obtener las categorías, códigos y subcódigos que presentamos para conocer la presencia de lo específico, interdisciplinar y sus formas de aproximación analítica y metodológica.

\section{Resultados}

\subsection{Palabras clave}

Los resultados obtenidos provienen de una selección inicial de la que hemos obtenido una serie de mapas, mostrando uno de ellos (Figura 1). El primer mapa, de máxima generalidad, muestra los elementos más nombrados, que hacen referencia a un perfil docente basado en la disciplina de la música como materia de contenido, así como diferentes géneros musicales, aspectos de la formación, el aprendizaje y la evaluación de música, quedando las temáticas más sistemáticos y estructurales de la música en un plano menos visible.

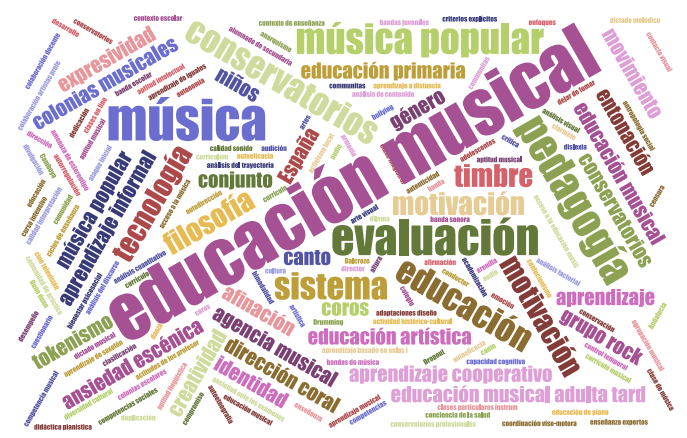

Fig. 1 Nube de palabras

Font: propia 


\subsubsection{Lo específico}

Los resultados destacados han sido: Música Popular y Jazz (7\%), Formación de Profesores de Música (5\%), Etapas y niveles de la educación musical (3\%), Música Instrumental (3\%) y Lenguaje Musical (2\%). La Música Popular abarca desde la música rock hasta distintas formas populares del s. XX, las tradicionales y el jazz. En cuanto a la Educación Musical, supone un gran conjunto que incluye temáticas específicas de todos los niveles de la educación formal e interesándose también por la no formal e informal.

\subsubsection{Lo interdisciplinar}

Este apartado requiere un análisis detallado. La interdisciplinariedad es una de las grandes apuestas del sistema por competencias que, según la OCDE (2005), responden a demandas complejas, utilizando y movilizando recursos psicosociales en un contexto particular. Si hablamos de la presencia de la música en la vida de las personas, su presencia multidisciplinar es uno de los grandes indicadores del valor de la música en lo social, descriptor de intereses y vehículo de acciones educativas para el desarrollo de múltiples aprendizajes y sus aproximaciones. Igualmente evidencia cómo la música amplía su radio de acción a otros espacios educativos y perfiles de lectores que las descubren desde otras disciplinas.

\subsubsection{Metodología y análisis científico por categorías}

Los resultados por categorías en referencia a totales y de la propia categoría han sido: Aproximaciones (3\%, 43\%; técnicas, instrumentos y medidas (1\%, 11\%); Instrumentos (11\%); Análisis cuantitativo $(1 \%, 18 \%)$; Análisis cualitativo $(1 \%, 14 \%)$; Metodologías mixtas $(0 \%, 4 \%)$. Su distribución por categorías es la siguiente: 1) Aproximaciones analíticas $(21 \%)$ : referencia a diferentes metodologías $(14 \%)$, estudio de caso $(7 \%) ; 2$ ) Técnicas, instrumentos y medidas $(21 \%)$ : a pesar de la gran dispersión destacan con un (4\%) Medición, Clasificación y Observación.; 3) Análisis cuantitativo (18\%): ocupan los lugares más destacados: validación de instrumentos, análisis factorial, quasi- experimento, registro todos con $(4 \%)$; 4) Análisis cualitativo (18\%): lo ocupan por igual con (4\%) análisis visual, narrativo, autoetnografía y etnografía. Y 5) Metodologías mixtas: (1\%): análisis de contenido y análisis del discurso 


\subsection{Líneas editoriales}

Los apartados de las web de las revistas son indicadores de calidad y, como tales, pueden dividirse en indicadores de estructura, focalizados en la normalización editorial, y los de procesos, parámetros de gestión, visibilidad y revisión por pares. Entre los indicadores de resultados aparecen el número de acceso, artículos descargados y número de citas bibliográficas de los artículos entre otros. En cuanto a temáticas, todas tienen en común por definición pertenecer al campo de la Educación Musical. Para conocerlas con detalle hemos seleccionado los sustantivos obteniendo 337 palabras y 189 códigos. El mapa mental muestra en la Figura 2 sus temáticas destacadas.

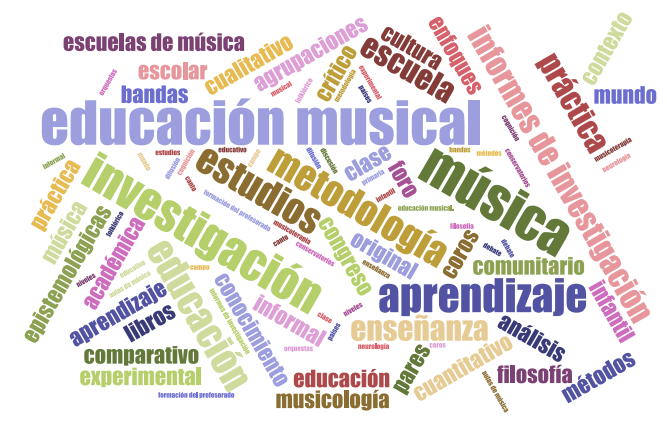

Fig. 2: Temáticas de las líneas editoriales

Font: Propia

\subsection{Agrupaciones y comparaciones}

\subsubsection{Agrupaciones temáticas}

Agrupando los resultados de códigos y categorías en niveles de máxima generalidad obtenemos cuatro grandes temáticas, aproximándonos así a nuestro objetivo final. Se trata de Formación, Práctica Educativa, Reflexión, Investigación y Difusión como indicadores comparables para la verificación de nuestra hipótesis. Así hemos llegado al tercer análisis de contenido que ha sido el que nos ha conducido a la meta. Las comparaciones entre LE y PC nos han dado el índice de intereses y tendencias en nuestro campo. Se han estudiado sus coincidencias y ausencias 


\subsubsection{Coincidencias.}

Los resultados más destacados corresponden a la educación musical multicultural y artística, a los que siguen la educación de adultos, profesores de música, valores, educación auditiva, formal, inclusiva y jazzística.

\subsubsection{Intereses}

Agrupando los temas llegamos a cuatro intereses de la Educación Musical en Líneas Editoriales, Palabras Clave y revistas: Investigación y difusión, Educación, Didáctica y Formación, Contenidos Musicales y Práctica Musical (Figura 3)

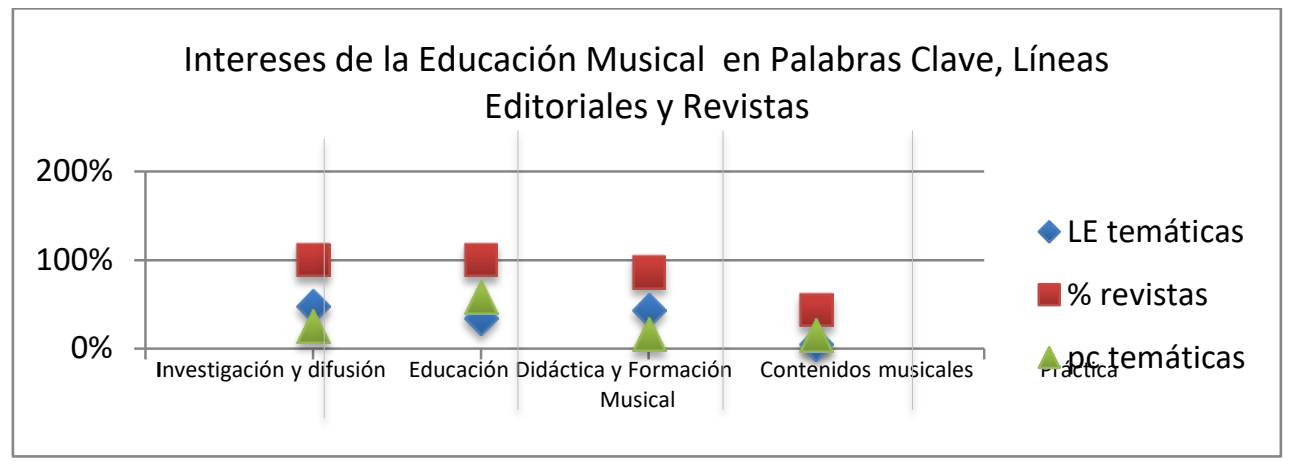

Fig. 3: Intereses de la Educación Musical en revistas y artículos

Font: Propia

\subsubsection{Tendencias}

El estudio de tendencias, mostrado en sus líneas de LE, PC y R ofrece los siguientes resultados

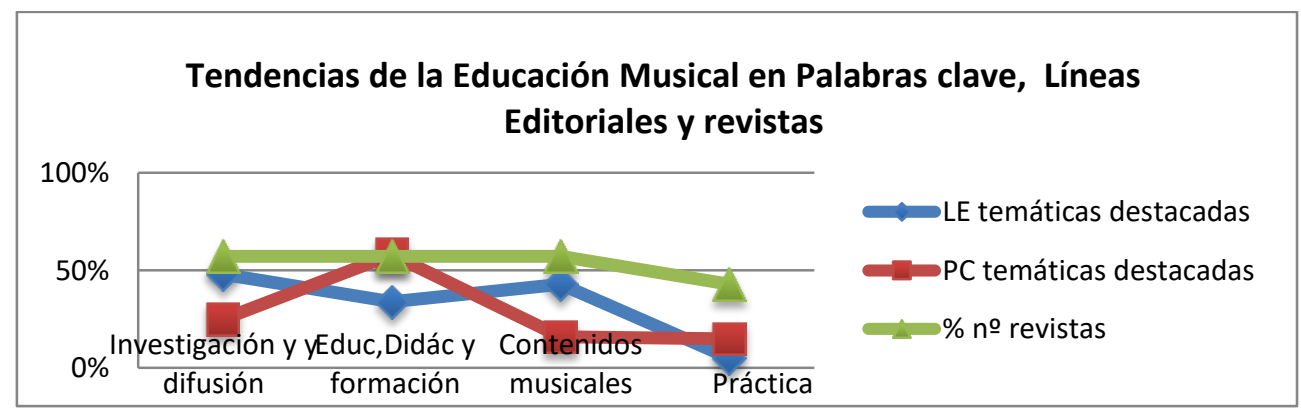

Fig. 4: Tendencias

Font: Propia 


\section{Conclusiones y discusión}

El estudio de los artículos y revistas de Educación Musical, por medio del análisis de contenido, nos ha permitido conocer cuáles son los intereses y tendencias del campo. La selección de las PC de los artículos y las LE de las revistas seleccionadas ha sido valiosas como indicadores de los intereses de autores y editores de la Educación Musical. El estudio de las palabras ha dado cuenta de qué es y cómo se estudia lo específico, interdisciplinar y metodológico. Las Líneas Editoriales marcan los límites, pero los autores crean los contenidos. Las coincidencias, discrepancias y ausencias en cada una y entre ellas proporcionan datos de mucho interés para la Educación Musical actual, y lo que es más importante, van anticipando su futuro mediante las tendencias que se van dibujando desde el aquí y ahora.

\section{Referencias}

ISO 2788, 1986 (E) Documentation - Guidelines for the establishment and development of monolingual thesauri. isiep.i. (http://vocabularies.unesco.org/browser/thesaurus/es/)

Martínez Tamayo, A. M., Ristuccia, C., Stubbs, E. A., Valdéz, J., Gamba, V. L., Mendes, P. V., ... \& Caminotti, M. (2011). La estructura sistemática del tesauro: indicadores para evaluar su calidad. Revista española de documentación científica,34, Madrid. DOI: 10.3989/redc.2011.1.765

OCDE (2005). Definition and Selection of Key Competencies: Executive Summary. Consultado el 12 de septiembre, 2018, en http://www.portal stat.admin.ch/deseco/news.htm

Porta, A. (2017). Conocer el entorno social de la música, una condición necesaria en la Educación Musical postmoderna. Dedica. Revista de Educação e Humanidades, (12), 69-82.

Porta, A. 2016) La competencia comunicativa en educación musical. Definición, presencia y repercusiones. III Congreso Nacional de Conservatorios superiores de Música y I Congreso Internacional de Conservatorios de Música. San Sebastián

Porta, A. (2007). Músicas públicas, escuchas privadas: Hacia una lectura de la música popular contemporánea (Vol. 20).: Universitat de València

Sloboda, J. A. (1985). The musical mind: The cognitive psychology of music. Oxford University Press

Strauss, A., \& Corbin, J. M. (1990). Basics of qualitative research: Grounded theory procedures and techniques. Sage Publications, Inc.

Talens, J., Romera, J., Tordera, A., \& Hernández Esteve, V. (1978). Elementos para una semiótica del texto artístico. Cátedra, Madrid, 1 . 
Tesauro de la Unesco: Lista estructurada de descriptores para la indización y la recuperación bibliográficas en las esferas de la educación, la ciencia, las ciencias sociales, la cultura y la comunicación (1984). París: Unesco 